\title{
Longer-term quality of life following major trauma: age only significantly affects outcome after the age of 80 years
}

This article was published in the following Dove Press journal:

Clinical Interventions in Aging

\author{
Thomas Gross' \\ Sabrina Morell' \\ Felix Amsler ${ }^{2}$ \\ 'Trauma Unit, Department of \\ Surgery, Kantonsspital Aarau, Aarau, \\ Switzerland; ${ }^{2}$ Amsler Consulting, \\ Basel, Switzerland
}

\begin{abstract}
Aim: Against the background of conflicting data on the topic, this study aimed to determine the differences in longer-term patient outcomes following major trauma with regard to age.

Materials and methods: A prospective trauma center survey of survivors of trauma ( $\geq 16$ years) was carried out employing a New Injury Severity Score (NISS) $\geq 8$ to investigate the influence of age on working capacity and several outcome scores, such as the trauma medical outcomes study Short Form-36 (physical component [PCS] and mental component [MCS]), the Euro Quality of Life (EuroQoL), or the Trauma Outcome Profile (TOP) at least 1 year following injury. Chi square tests, $t$-tests, and Pearson correlations were used as univariate; stepwise regression as multivariate analysis. Significance was set at $p<0.05$.

Results: In all, 718 major trauma patients (53.4 \pm 19.4 years; NISS 18.4 \pm 9.2$)$ participated in the study. Multivariate analysis showed only low associations of patient or trauma characteristics with longer-term outcome scores, highest for the Injury Severity Score of the extremities with the PCS $\left(R^{2}=0.08\right)$ or the working capacity of employed patients $\left(n=383 ; R^{2}=0.04\right)$. For age, overall associations were even lower (best with the PCS, $\mathrm{R}^{2}=0.04$ ) or could not be revealed at all (TOP or MCS). Subgroup analysis with regard to decennia revealed the age effect to be mainly attributable to patients aged $\geq 80$, who presented with a significantly worse outcome compared to younger people in all overall and physical component scores $(p<0.001)$. In patients under 80 years an association of age was only found for EuroQoL $\left(R^{2}=0.01\right)$ and the PCS $\left(\mathrm{R}^{2}=0.03\right)$.
\end{abstract}

Conclusion: Given the small impact of age on the longer-term outcomes of major trauma patients, at least up to the age of 80 years, resuscitation as well as rehabilitation strategies should be adapted accordingly.

Keywords: major trauma, longer term, outcome, quality of life, working capacity, age

\section{Introduction}

In the discussion about avoiding unnecessary efforts and expenditures following major trauma, for example, with regard to triage in the field ${ }^{1,2}$ or specific rehabilitation efforts, the argument of a limited outcome in the elderly is often heard. However, most studies cited only reports on parameters of short-term outcome, such as morbidity and mortality. ${ }^{3}$ In contrast, specific challenges of any treatment in older age, such as increasing frailty and loss of independence, become more and more evident in the literature. ${ }^{4-6}$ In addition, with regard to longer-term aspects, patient-centered, health-related outcomes are increasingly recognized as an important benchmark. ${ }^{7,8}$ As no formally agreed definition of quality of life (QoL) exists, ${ }^{9}$ various measures used in the context of QoL, health status, functional status, or health-related quality of life (HRQoL) may
Correspondence: Thomas Gross Trauma Unit, Department of Surgery, Kantonsspital Aarau, Tellstrasse I, $\mathrm{CH}-500 \mathrm{I}$ Aarau, Switzerland

Tel +4I 62838660 I

Fax +4I 628384847

Email thomas.gross@ksa.ch 
reflect different aspects of an individual's well-being ${ }^{10,11}$ and may result in seemingly inconsistent findings in the literature.

Only a few studies report on the differences in the longer-term outcomes of the elderly vs younger survivors of major trauma, presenting conflicting results ranging from decreasing longer-term outcomes with increasing age or in the elderly to no association of outcome scoring with age. ${ }^{12-16}$ In addition, the definition of elderly in the literature varies widely, most of them using an age of at least 55 or 65 years as the cutoff. ${ }^{3,17}$

Against this background, we were interested in the longer-term outcomes of adult patients following treatment for major trauma in a Swiss trauma center, asking the specific question of to what extent HRQoL and functional outcome as measured on validated scores as well as working capacity are actually associated with age. To focus on the hypothesis that longer-term outcome is reduced in elderly patients we particularly investigated different aspects of outcome across different age groups. Multiple well-accepted and newly developed measures of outcome were used simultaneously in this investigation to look specifically at the broad spectrum of longer-term outcome and, more generally, to evaluate comparatively the possibly divergent findings between individual instruments.

\section{Materials and methods}

The survey was conducted at a Swiss trauma center with a catchment population of about 750,000 inhabitants. Within this prospective quality controlled study (NCT02165137), approved by the regional ethics committee (Cantonal Ethical Commission Aargau), all consecutive major trauma patients (New Injury Severity Score $[\text { NISS }]^{18} \geq 8$ ) who arrived and were treated at the emergency department from January 1, 2010 to December 31, 2015 within 24 hours of the accident were recruited for longer-term follow-up. Hospital procedural guidelines followed international standards. ${ }^{19,20}$ The investigation included all survivors of trauma aged $>15$ years at the time of the accident who had participated in at least one of the two follow-up time points after injury. All participants provided written informed consent. Exclusion criteria for this survey were patients under the age of 16 , those with an NISS $<8$, deceased, or those presenting with a Glasgow Outcome Scale $(\mathrm{GOS})^{21}$ value of 2 (persistent vegetative state) at follow-up. For the analysis of working capacity, only patients aged under 64 at the time of the accident were included. Figure 1 demonstrates the follow-up flow chart of the investigation.

\section{Data management}

Data management was executed by specifically trained study nurses who were not involved in the treatment

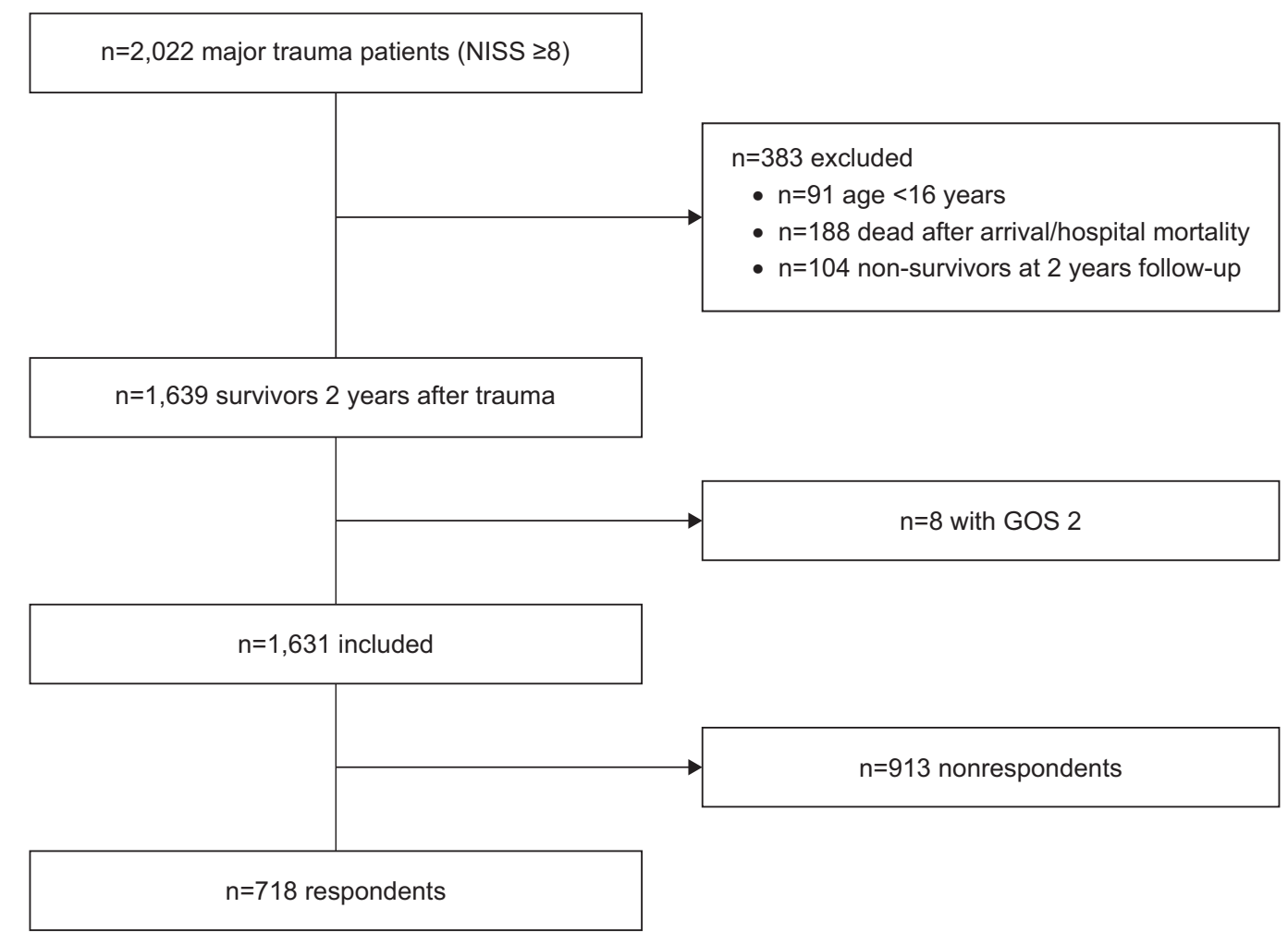

Figure I Flow chart of selection of eligible patients.

Abbreviations: NISS, New Injury Severity Score; GOS, Glasgow Outcome Scale. 
of single cases. Injury severity was determined based on the maximum information available at the end of hospitalization. Demographic characteristics included age at the time of injury (years), gender (male/female), and nationality (Swiss yes/no). Injury-related variables were trauma energy graded as high (traffic accident, fall from $>3 \mathrm{~m}$ or a shot) or low (all other cases), mono- vs multiple injury (Abbreviated Injury Scale [AIS] $>0$ in at least two body regions ${ }^{16}$ ), the Glasgow Coma Scale (GCS; first available value), ${ }^{22}$ the Mechanism, GCS, Age, and Arterial score, ${ }^{23}$ and the Simplified Acute Physiology Score II predicted mortality $;{ }^{24}$ the AIS, ${ }^{25}$ the Injury Severity Score (ISS), ${ }^{26}$ and NISS following the version 2005, update 2008 of the Trauma Register of the German Trauma Society (DGU ${ }^{\circledR} ;$ http://www.traumaregister-dgu.de), with maximum information at the end of hospitalization and with special combinations for subgroup analysis such as the total NISS value minus the AIS head for the trauma severity of single patients without traumatic brain injury; and expected riskadjusted mortality measured with the Revised Injury Severity Classification 2 (RISC 2). ${ }^{27}$ Treatment-related variables included need for emergency intubation (yes/no), emergency operation (within 4 hours after hospital admittance following the TraumaRegister $\mathrm{DGU}^{\circledR}$ criteria, yes/no), admittance to the intensive care unit (ICU, yes/no) and length of ICU stay, length of hospital stay, and transfer to a rehabilitation clinic following initial hospital stay (yes/no).

The survival status of nonrespondents at the time of follow-up was obtained by contacting next of kin, family practitioners, and local registry offices. Patient longer-term outcomes were assessed 1 and 2 years following trauma by a postal survey, complemented by phone interviews for missing or dubious answers undertaken by study nurses not involved in the initial treatment of patients. If patients answered at both follow-up time points, the 1-year information was used for this evaluation. Standardized self-report questionnaires comprised a combination of validated QoL and functional scoring instruments with respect to outcome measurements. The follow-up questionnaires included the following standard instruments: GOS, ${ }^{21}$ Euro Quality of Life (EuroQoL) Group (http:// www.euroqol.org) HRQoL on five dimensions (EQ-5D), ${ }^{28}$ medical outcomes study Short Form-36 (SF-36, single dimensions and physical component [PCS] and mental component $[\mathrm{MCS}]),{ }^{29}$ the Trauma Outcome Profile (TOP, single dimensions and physical and mental component), ${ }^{11,30}$ and the Quality of Life after Brain Injury (QOLIBRI). ${ }^{31,32}$ In addition, patients were asked about several socioeconomic factors such as working capacity before injury and at the time of follow-up, educational level (1, only compulsory education;
2, professional qualification; 3 , university degree), and living status (living in a partnership yes/no). Official working capacity $(0 \%-100 \%$, as referred to a responsible doctor or insurance company) was given only for employed patients under the age of 64 years on the day of accident.

\section{Statistics}

Data are displayed as mean $\pm \mathrm{SD}$ for numeric variables or mean \pm CIs in figures. Numbers and percentages are given for nominal variables if not stated otherwise. All statistical tests were two-tailed and $p<0.05$ was considered significant. Differences of mean values were calculated with the Students $t$-test for independent samples, nominal variables were compared with chi square statistics. For univariate statistics, missing cases were excluded variable-wise. Documented variables suspected or known from the literature to be possible factors associated with outcome were first analyzed by univariate correlation testing to obtain Pearson's $r$. For multivariate regression analysis, age, gender, educational level, and living in a partnership as sociodemographics as well as AIS 1-AIS 6 and RISC 2 as injury-associated variables were entered stepwise with an entry criterion set at $p<0.05$. Data are given as beta, total $\mathrm{R}^{2}$, and $\mathrm{R}^{2}$ change; missing values were replaced by the mean of the whole cohort. Data were analyzed using IBM SPSS Statistics for Windows 24.0 (IBM Corporation, Armonk, NY, USA).

\section{Results}

In all, 718 survivors of major trauma participated in this longer-term survey. In 556 cases the 1-year and in 162 cases the 2-year follow-up questionnaire information were used for analysis (see follow-up chart, Figure 1). With regard to sociodemographics, trauma and treatment characteristics respondents did not differ from nonrespondents in major criteria such as mean age, gender, and overall trauma severity. On the other hand, respondents were more often Swiss, sustained high-energy trauma, and on average presented with less severe head and neck trauma (Table 1). Table 2 depicts the longer-term outcomes of major trauma patients as measured in single scores. The mean working capacity of employed patients under the age of 64 years $(n=383)$ was $96.2 \% \pm 15.9 \%$ before injury and $76.3 \% \pm 35.8 \%$ at longerterm follow-up $(p<0.001)$.

Table 3 gives univariate correlations of patient, trauma, and treatment characteristics with investigated outcome scores. Only low associations were found, highest for the trauma severity of injuries of the extremities with the bodily function in the SF-36 ( $r=-0.31)$. With regard to age, for example, no correlations were revealed with either the 
Table I Sociodemographics, trauma, and treatment characteristics of respondents vs nonrespondents of longer-term follow-up

\begin{tabular}{|c|c|c|c|}
\hline \multirow[t]{2}{*}{ Variable } & \multirow{2}{*}{$\begin{array}{l}\text { Nonrespondents } \\
(\mathrm{N}=913) \\
\text { Mean (SD) }\end{array}$} & \multirow{2}{*}{$\begin{array}{l}\text { Respondents } \\
(\mathrm{N}=718) \\
\text { Mean (SD) }\end{array}$} & \multirow[t]{2}{*}{$p$-value } \\
\hline & & & \\
\hline $\begin{array}{l}\text { Age at time of injury } \\
\text { (years) }\end{array}$ & $54.05(22.17)$ & $53.44(19.35)$ & 0.560 \\
\hline GCS & I3.77 (2.65) & I3.57 (3.06) & 0.151 \\
\hline MGAP & $25.12(3.77)$ & $25.09(3.92)$ & 0.883 \\
\hline ISS & I3.60 (7.47) & I3.80 (7.49) & 0.580 \\
\hline NISS & $18.1 \mid(9.28)$ & $18.38(9.21)$ & 0.564 \\
\hline RISC 2 & $5.38(10.68)$ & $3.59(6.93)$ & $<0.001$ \\
\hline SAPS II & $26.71(12.79)$ & $26.99(11.22)$ & 0.767 \\
\hline AIS I head and neck & $\mathrm{I} .87(\mathrm{I} .55)$ & $1.69(1.53)$ & 0.023 \\
\hline AIS 2 face & $0.35(0.79)$ & $0.40(0.85)$ & 0.270 \\
\hline AIS 3 chest & $0.94(1.38)$ & $\mathrm{I} .07(\mathrm{I} .43)$ & 0.060 \\
\hline AIS 4 abdomen & $0.43(0.96)$ & $0.48(1.02)$ & 0.312 \\
\hline AIS 5 extremities & I.II (I.27) & $1.23(1.29)$ & 0.067 \\
\hline AIS 6 external & $0.36(0.57)$ & $0.38(0.56)$ & 0.475 \\
\hline $\begin{array}{l}\text { Length of hospital } \\
\text { stay (days) }\end{array}$ & $10.21(9.39)$ & $12.98(11.87)$ & $<0.001$ \\
\hline \multirow{2}{*}{$\begin{array}{l}\text { GOS (at hospital } \\
\text { discharge) }\end{array}$} & $4.8(0.46)$ & $4.85(0.40)$ & 0.013 \\
\hline & n (\%) & n (\%) & $p$-value \\
\hline Gender, female & $272(29.9)$ & $239(33.3)$ & 0.139 \\
\hline Swiss & $650(76.5)$ & $596(85.1)$ & $<0.001$ \\
\hline High-energy trauma & $400(44.4)$ & 391 (54.5) & $<0.001$ \\
\hline Patients aged $>63$ years & $351(38.5)$ & $258(35.9)$ & 0.283 \\
\hline $\begin{array}{l}\text { Need for emergency } \\
\text { operation }\end{array}$ & $56(6.1)$ & $53(7.4)$ & 0.322 \\
\hline ICU stay & $427(46.9)$ & $385(53.6)$ & 0.007 \\
\hline ISS $>15$ & $295(32.4)$ & $250(34.8)$ & 0.301 \\
\hline Multiply injured & $613(67.3)$ & $496(69.1)$ & $0.44 I$ \\
\hline $\begin{array}{l}\text { Rehabilitation clinic } \\
\text { following initial } \\
\text { hospital stay }\end{array}$ & $209(23.2)$ & $223(3 I . I)$ & $<0.001$ \\
\hline
\end{tabular}

Abbreviations: GCS, Glasgow Coma Scale; MGAP, Mechanism, Glasgow Coma Scale, Age, and Arterial score; ISS, Injury Severity Score; NISS, New Injury Severity Score; RISC 2, Revised Injury Severity Classification 2; SAPS II, Simplified Acute Physiology Score II; AIS, Abbreviated Injury Scale (eg, AIS I: head and neck etc); GOS, Glasgow Outcome Score; ICU, intensive care unit, multiply injured.

physical or the mental component of TOP, and the highest association was found with the PCS $(r=-0.29)$.

To further examine the possible impact of age on the longer-term outcome following major trauma, patients were stratified in single decennia groups. Figure 2 illustrates the poorer outcome of octogenarians and older patients vs all other age groups under investigation. Compared to younger people, outcome in those aged $\geq 80$ years was significantly worse $(p<0.001)$ in all overall (GOS, EuroQoL, QOLIBRI) and in physical component scores (SF-36, TOP), but not in mental components or social dimensions of scores (Table 2). For example, on the PCS patients aged $\geq 80$ years rated only $61 \%$ of function compared to younger patients. When 70 - to 79-year-old patients were compared with the younger ones, no statistically significant difference was found (data not shown).

Table 4 reveals the sociodemographics, trauma, and treatment characteristics of patients aged $\geq 80$ years vs younger patients. The group of at least octogenarians did not differ from younger patients in major criteria such as injury severity (both, overall and in single body regions), rate of admissions from another hospital, or length of hospital stay. Significant differences between groups were found for all age-dependent scores. In addition, the group of at least octogenarians included a higher percentage of Swiss and female patients, lower educational level, a lower percentage of high-energy trauma cases, a lower mean initial GCS following injury, and a higher percentage of patients transferred to a rehabilitation clinic following initial hospital stay.

When univariate data for age with longer-term outcome scores were compared across the group of all patients and only those under the age of 80 years (first vs second column in Table 3), maximum low correlations in both groups for all overall and physical component scores were found to be even lower in patients $<80$ years of age.

Multivariate analysis (Table 5) confirmed this finding: in the group of all patients the majority of outcome scores correlated best with the injury severity of the extremities (AIS 5), for example in the PCS $\left(\mathrm{R}^{2}=0.08\right)$, or the EuroQoL $\left(\mathrm{R}^{2}=0.06\right.$; both $p<0.001)$. The same was valid for the working capacity of employed patients only $\left(\mathrm{n}=383 ; \mathrm{R}^{2}=0.05, p<0.001\right)$. The mental components of the SF-36 and the TOP and the total QOLIBRI demonstrated best correlations with the education level of patients. No or lower associations with longer-term outcome were revealed for age than for injury severity and education level, at best in the PCS $\left(\mathrm{R}^{2}\right.$ change $\left.=0.06\right)$. In patients aged $<80$ years, a significant association of age was only found in the EuroQoL $\left(\mathrm{R}^{2}\right.$ change $\left.=0.01\right)$ and the PCS $\left(R^{2}\right.$ change $\left.=0.03\right)$, but not for any of the other outcome scores investigated.

\section{Discussion}

To the best of our knowledge, this prospective study is the first to evaluate in a comparative manner several health-related and functional outcome scores as well as working capacity of adult patients 1 or 2 years following injury. A definitive impact of age on the longer-term outcome of survivors of major trauma was only found in at least octogenarians. For example, patients aged $\geq 80$ years in the PCS rated $<2 / 3$ of the function compared to younger patients. In contrast, multivariate analysis of patients $<80$ years revealed no significant association of age with longer-term outcome as 
Table 2 Longer-term outcome of major trauma patients in single scores

\begin{tabular}{|c|c|c|c|c|}
\hline \multirow[t]{2}{*}{ Variable } & \multirow{2}{*}{$\frac{\text { Total }(\mathbf{N}=7 \mid 8)}{\text { Mean }(\mathrm{SD})}$} & \multirow{2}{*}{$\frac{<80 \text { years }(\mathrm{N}=659)}{\text { Mean }(\mathrm{SD})}$} & \multirow{2}{*}{$\frac{\geq 80 \text { years }(\mathrm{N}=59)}{\text { Mean }(\mathrm{SD})}$} & \multirow{2}{*}{$\frac{\text { Difference }}{p \text {-value }}$} \\
\hline & & & & \\
\hline SF-36 physical functioning & $72.36(29.10)$ & $74.73(27.90)$ & $46.02(29.37)$ & $<0.001$ \\
\hline SF-36 role physical & $57.38(43.08)$ & $59.82(42.73)$ & 30.51 (37.73) & $<0.001$ \\
\hline SF-36 bodily pain & $66.83(27.50)$ & $67.4 I(27.31)$ & $60.34(28.96)$ & 0.059 \\
\hline SF-36 general health & $65.66(21.04)$ & $66.17(21.15)$ & $59.93(19.02)$ & 0.032 \\
\hline SF-36 vitality & $56.65(21.26)$ & $57.31(21.36)$ & $49.14(18.66)$ & 0.005 \\
\hline SF-36 social functioning & 78.5। (25.95) & $79.25(25.60)$ & $70.34(28.5 \mathrm{I})$ & 0.011 \\
\hline SF-36 role emotional & $70.60(41.50)$ & $71.57(4 \mid .32)$ & $59.89(42.34)$ & 0.038 \\
\hline SF-36 mental health & $71.49(20.11)$ & $71.62(20.26)$ & $70.00(18.36)$ & 0.557 \\
\hline SF-36 PCS & $45.90(10.38)$ & $46.56(10.18)$ & $38.31(9.73)$ & $<0.001$ \\
\hline SF-36 MCS & $47.34(12.94)$ & 47.40 (12.99) & $46.63(12.45)$ & 0.673 \\
\hline TOP depression & $82.62(22.4 I)$ & $82.83(22.52)$ & $80.26(21.20)$ & 0.402 \\
\hline TOP anxiousness & $85.19(20.01)$ & $85.07(20.35)$ & 86.49 (I5.78) & 0.606 \\
\hline TOP PTSD & 79.11 (22.82) & $79.06(23.18)$ & $79.68(|8.3|)$ & 0.843 \\
\hline TOP social interaction & 80.14 (25.09) & $79.97(25.38)$ & $82.08(21.55)$ & 0.539 \\
\hline TOP daily activities & $86.09(21.60)$ & 87.89 (19.97) & $66.14(28.25)$ & $<0.001$ \\
\hline TOP mental functioning & $70.05(26.83)$ & 71.11 (26.76) & $58.19(24.79)$ & $<0.001$ \\
\hline TOP body image & $89.90(22.49)$ & $89.54(23.23)$ & $93.81(10.84)$ & 0.162 \\
\hline TOP satisfaction & $87.22(25.98)$ & $87.11(26.27)$ & $88.43(22.72)$ & 0.708 \\
\hline TOP pain before injury & $90.67(15.53)$ & 91.47 (14.92) & $81.81(19.10)$ & $<0.001$ \\
\hline TOP function before injury & $93.13(13.92)$ & $93.94(13.23)$ & $84.19(17.90)$ & $<0.001$ \\
\hline TOP pain longer term & $82.42(20.69)$ & $82.96(20.60)$ & $76.50(21.01)$ & 0.022 \\
\hline TOP function longer term & $83.94(21.08)$ & $84.60(21.12)$ & $76.68(19.4 I)$ & 0.006 \\
\hline TOP development pain & $-8.26(19.59)$ & $-8.53(19.79)$ & $-5.31(17.07)$ & 0.227 \\
\hline \multicolumn{5}{|l|}{ (neg $=$ worse at longer term) } \\
\hline TOP development function & $-9.20(19.88)$ & $-9.36(20.18)$ & $-7.52(16.23)$ & 0.496 \\
\hline \multicolumn{5}{|l|}{ (neg $=$ worse at longer term) } \\
\hline TOP number of conspicuous dimensions & $2.99(2.78)$ & $2.89(2.80)$ & $4.12(2.37)$ & 0.001 \\
\hline TOP physical component & $85.62(16.27)$ & $86.27(16.18)$ & $78.44(15.74)$ & $<0.001$ \\
\hline TOP mental component & $80.63(18.63)$ & $80.76(18.93)$ & 79.15 (14.89) & 0.530 \\
\hline QOLIBRI cognition & 75.76 (23.49) & $76.64(23.04)$ & $66.04(26.25)$ & 0.001 \\
\hline QOLIBRI self & 73.89 (20.77) & $74.08(20.87)$ & $71.90(19.76)$ & $0.44 I$ \\
\hline QOLIBRI daily life and autonomy & $78.26(23.25)$ & $79.58(22.37)$ & $63.75(27.6 I)$ & $<0.001$ \\
\hline QOLIBRI social relationships & $78.58(20.24)$ & $78.44(20.50)$ & $80.10(17.04)$ & 0.550 \\
\hline QOLIBRI emotions & $84.87(20.37)$ & $84.99(20.30)$ & $83.48(21.30)$ & 0.588 \\
\hline QOLIBRI physical problems & $76.10(22.65)$ & $77.61(21.73)$ & $59.15(25.89)$ & $<0.001$ \\
\hline QOLIBRI total & $77.35(18.66)$ & 78.07 (I8.47) & $69.45(19.02)$ & $<0.001$ \\
\hline EuroQoL & $0.73(0.21)$ & $0.74(0.21)$ & $0.6 I(0.2 I)$ & $<0.001$ \\
\hline GOS & $4.77(0.49)$ & $4.79(0.46)$ & $4.53(0.73)$ & $<0.001$ \\
\hline
\end{tabular}

Abbreviations: SF-36, trauma medical outcomes study Short Form-36; PCS, physical sum component score; MCS, mental sum component score, and single SF-36 domains; TOP, Trauma Outcome Profile, physical and mental component, and single dimensions; PTSD, posttraumatic stress disorder; QOLIBRI, Quality of Life after Brain Injury total score and single dimensions; EuroQoL, Euro Quality of Life Group HRQoL on five dimensions; GOS, Glasgow Outcome Scale.

measured on the GOS, the TOP, the QOLIBRI, or the MCS. The associations with age found in patients $<80$ years on the EuroQoL and the PCS were small and even lower than the already low associations of longer-term outcome found with injury severity or education level.

Holbrook et al were one of the first authors to describe a reduced outcome with increasing age 12 and 18 months after major trauma (mean ISS 18), using the quality of well-being scale only. ${ }^{12}$ Other authors reported on at least partly lower outcomes, mostly with regard to physical dimensions, for example, after the age of 55 years at mean 2.8 years following severe trauma (ISS $>15$ ) on the World Health Organization
Quality of Life assessment instrument-BREF ${ }^{13}$ or even in the age category of 31-55 years compared to younger people following major trauma (mean ISS 14) in several dimensions of the SF-36. ${ }^{14}$ A recent study by Gunning et al on the longterm outcome of a trauma level I center cohort (mean ISS 13) 5-10 years after injury, using the SF-36 and the EQ-5D, found in comparison to two younger age groups a lower HRQoL according to physical function in the group of eldest patients ( $>70$ years). ${ }^{8}$ A retrospective long-term follow-up in 147 patients at a mean of 6 years after polytrauma (mean ISS 28) reported a univariate correlation of age with the EuroQoL, both the PCS and the MCS, and the mental but not the physical 
Table 3 Univariate correlations of patient, trauma, and treatment characteristics with investigated outcome data ( $N=7 / 8$, working capacity $\mathrm{N}=383$ )

\begin{tabular}{|c|c|c|c|c|c|c|c|c|c|c|}
\hline Pearson $r$ & 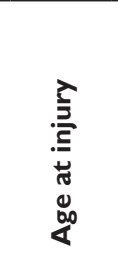 & 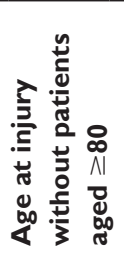 & 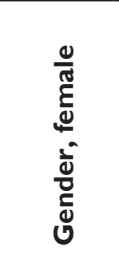 & 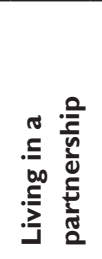 &  & $\underline{\underline{\Lambda}}$ & $\frac{\varkappa}{z}$ & 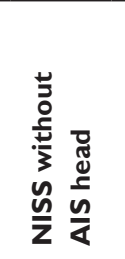 & 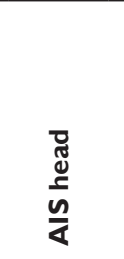 & 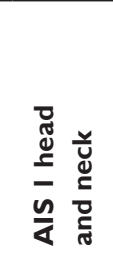 \\
\hline SF-36 physical functioning & $-0.29 * * *$ & $-0.20 * * *$ & $-0.08^{*}$ & 0.03 & $0.14 * * *$ & $-0.09 *$ & -0.05 & $-0.17 * * *$ & $0.17 * * *$ & $0.17 * * *$ \\
\hline SF-36 role physical & $-0.23 * * *$ & $-0.16 * * *$ & -0.07 & 0.03 & $0.12 * * *$ & $-0.11 * *$ & $-0.11 * *$ & $-0.15^{* * *}$ & 0.05 & 0.04 \\
\hline SF-36 bodily pain & -0.03 & 0.01 & -0.01 & 0.03 & $0.14 * * *$ & -0.05 & -0.01 & $-0.10^{* *}$ & $0.12^{* * *}$ & $0.12 * *$ \\
\hline SF-36 general health & $-0.12^{* * *}$ & $-0.10^{*}$ & 0.03 & -0.02 & $0.13 * * *$ & -0.06 & -0.03 & -0.07 & 0.04 & 0.03 \\
\hline SF-36 vitality & -0.03 & 0.03 & $-0.14 * * *$ & 0.03 & $0.16 * * *$ & -0.02 & -0.04 & -0.03 & -0.01 & -0.02 \\
\hline SF-36 social functioning & $-0.08^{*}$ & -0.04 & -0.04 & 0.00 & $0.14 * * *$ & $-0.08 *$ & $-0.11 * *$ & $-0.09 *$ & -0.05 & -0.05 \\
\hline SF-36 role emotional & $-0.14 * * *$ & $-0.13^{* * *}$ & -0.06 & $0.08 *$ & $0.17 * * *$ & $-0.10 * *$ & $-0.11 * *$ & -0.06 & $-0.08 *$ & $-0.08^{*}$ \\
\hline SF-36 mental health & 0.02 & 0.04 & -0.07 & 0.01 & $0.21 * * *$ & -0.01 & -0.02 & 0.00 & -0.03 & -0.03 \\
\hline SF-36 PCS & $-0.23 * * *$ & $-0.15^{* * *}$ & -0.03 & 0.01 & $0.10 * *$ & $-0.09 *$ & -0.04 & $-0.17 * * *$ & $0.18^{* * *}$ & $0.18^{* * *}$ \\
\hline SF-36 MCS & 0.00 & 0.01 & -0.07 & 0.03 & $0.19 * * *$ & -0.05 & $-0.10 *$ & -0.01 & $-0.13 * * *$ & $-0.13^{* * *}$ \\
\hline TOP depression & 0.04 & 0.07 & -0.02 & $0.08 *$ & $0.20 * * *$ & -0.06 & -0.07 & -0.06 & -0.03 & -0.03 \\
\hline TOP anxiety & 0.02 & 0.02 & $-0.09 *$ & 0.00 & $0.17 * * *$ & -0.07 & -0.05 & 0.00 & -0.06 & -0.07 \\
\hline TOP PTSD & $0.13 * * *$ & $0.14 * * *$ & $-0.12 * * *$ & 0.02 & $0.20 * * *$ & -0.01 & 0.02 & 0.00 & 0.01 & 0.01 \\
\hline TOP social aspects & 0.03 & 0.02 & -0.01 & 0.04 & $0.19 * * *$ & $-0.10 * *$ & $-0.10 * *$ & $-0.07^{*}$ & -0.06 & -0.06 \\
\hline TOP daily activities & $-0.27 * * *$ & $-0.16 * * *$ & 0.00 & 0.03 & $0.17 * * *$ & -0.03 & -0.02 & $-0.09 *$ & $0.10 * *$ & $0.09 *$ \\
\hline TOP mental functioning & $-0.10 * *$ & -0.04 & -0.03 & -0.03 & $0.15^{* * *}$ & $-0.14 * * *$ & $-0.14 * * *$ & -0.03 & $-0.18 * * *$ & $-0.18 * * *$ \\
\hline TOP body image & $0.22 * * *$ & $0.22 * * *$ & -0.01 & 0.07 & $0.11 * *$ & $-0.09 *$ & $-0.08^{*}$ & $-0.13 * * *$ & 0.06 & 0.06 \\
\hline TOP satisfaction & 0.00 & -0.01 & 0.01 & -0.02 & $0.10 * *$ & -0.07 & $-0.09 *$ & $-0.09 *$ & -0.03 & -0.01 \\
\hline TOP pain before & $-0.22 * * *$ & $-0.17 * * *$ & -0.05 & 0.05 & $0.09 *$ & 0.04 & 0.05 & 0.06 & 0.00 & -0.02 \\
\hline $\begin{array}{l}\text { TOP physical functioning } \\
\text { before injury }\end{array}$ & $-0.24 * * *$ & $-0.17 * * *$ & -0.04 & 0.02 & $0.08^{*}$ & 0.06 & $0.08^{*}$ & 0.05 & 0.05 & 0.03 \\
\hline TOP pain after injury & -0.03 & 0.02 & -0.01 & 0.02 & $0.16 * * *$ & -0.02 & 0.01 & -0.04 & 0.07 & 0.06 \\
\hline $\begin{array}{l}\text { TOP physical functioning } \\
\text { after injury }\end{array}$ & $-0.09 *$ & -0.04 & -0.03 & 0.02 & $0.14 * * *$ & $-0.08^{*}$ & -0.05 & $-0.10^{* *}$ & 0.05 & 0.03 \\
\hline $\begin{array}{l}\text { TOP development pain } \\
\text { (neg = worse at longer term) }\end{array}$ & $0.15 * * *$ & $0.15 * * *$ & 0.03 & -0.01 & $0.10 * *$ & -0.06 & -0.03 & $-0.09 *$ & 0.07 & 0.07 \\
\hline $\begin{array}{l}\text { TOP development function } \\
\text { (neg = worse at longer term) }\end{array}$ & 0.07 & 0.07 & -0.01 & 0.01 & $0.09 *$ & $-0.13^{* * *}$ & $-0.11 * *$ & $-0.14 * * *$ & 0.02 & 0.01 \\
\hline TOP physical component & 0.05 & -0.01 & 0.06 & -0.02 & $-0.21^{* * *}$ & 0.07 & 0.05 & 0.07 & -0.01 & -0.01 \\
\hline TOP mental component & -0.05 & 0.02 & -0.02 & 0.05 & $0.19 * * *$ & $-0.08^{*}$ & -0.05 & $-0.12 * *$ & $0.09 *$ & $0.08 *$ \\
\hline TOP depression & 0.02 & 0.04 & -0.05 & 0.02 & $0.21 * * *$ & $-0.10 * *$ & $-0.10^{*}$ & -0.05 & $-0.08^{*}$ & $-0.08^{*}$ \\
\hline$Q$ cognition & $-0.15^{* * *}$ & $-0.10^{* *}$ & 0.01 & 0.00 & $0.20 * * *$ & -0.06 & $-0.08^{*}$ & 0.02 & $-0.14 * * *$ & $-0.14 * * *$ \\
\hline$Q$ self & -0.01 & 0.01 & -0.07 & 0.00 & $0.13 * * *$ & -0.06 & -0.07 & -0.05 & -0.02 & -0.04 \\
\hline $\mathrm{Q}$ daily life and autonomy & $-0.15^{* * *}$ & -0.07 & -0.02 & 0.02 & $0.19 * * *$ & $-0.09 *$ & $-0.10 * *$ & $-0.10^{* *}$ & -0.01 & -0.02 \\
\hline Q social relationships & -0.02 & -0.03 & 0.01 & $0.09 *$ & $0.12 * * *$ & -0.05 & -0.05 & -0.04 & -0.02 & -0.03 \\
\hline$Q$ emotions & 0.04 & 0.06 & -0.03 & 0.06 & $0.18^{* * *}$ & -0.07 & -0.06 & -0.04 & -0.04 & -0.05 \\
\hline Q physical problems & $-0.21 * * *$ & $-0.12^{* *}$ & -0.06 & 0.03 & $0.14 * * *$ & $-0.11 * *$ & $-0.09 *$ & $-0.13 * * *$ & 0.02 & 0.01 \\
\hline QOLIBRI total & $-0.12^{* * *}$ & -0.06 & -0.03 & 0.02 & $0.20 * * *$ & $-0.08^{*}$ & $-0.08^{*}$ & -0.04 & -0.06 & -0.07 \\
\hline EuroQoL & $-0.15^{* * *}$ & $-0.08^{*}$ & -0.03 & 0.03 & $0.17 * * *$ & -0.07 & -0.04 & $-0.12^{* * *}$ & $0.10 * *$ & $0.10 *$ \\
\hline GOS longer term & $-0.14 * * *$ & $-0.08^{*}$ & 0.00 & -0.02 & $0.09 *$ & $-0.12^{* *}$ & $-0.13^{* * *}$ & $-0.15^{* * *}$ & 0.00 & 0.00 \\
\hline Working capacity & $-0.13^{*}$ & $-0.13^{*}$ & 0.00 & -0.01 & $0.14 * *$ & $-0.17 * *$ & $-0.11 *$ & -0.10 & -0.07 & -0.07 \\
\hline
\end{tabular}

Notes: $* p<0.05 ; * * p<0.01 ; * * * p<0.001$.

Abbreviations: SF-36, trauma medical outcomes study Short Form-36; PCS, physical sum component score; MCS, mental sum component score, and single domains; TOP, Trauma Outcome Profile, physical and mental component and single dimensions; PTSD, posttraumatic stress disorder; Q, QOLIBRI, Quality of Life after Brain Injury total score and single dimensions; EuroQoL, Euro Quality of Life Group HRQoL on five dimension; GOS longer term, Glasgow Outcome Score at longer-term follow-up. 


\begin{tabular}{|c|c|c|c|c|c|c|c|c|c|c|c|c|}
\hline 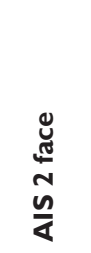 & 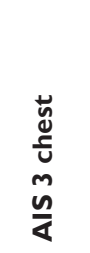 & 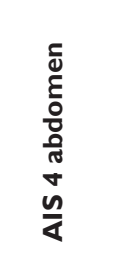 & 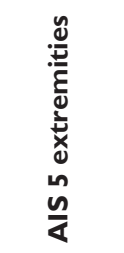 & 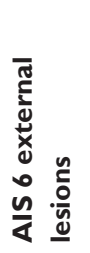 & $\underset{u}{\tilde{u}}$ & $\bigcup_{\mathcal{U}}^{n}$ & 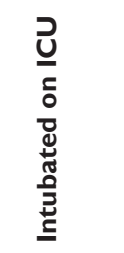 & $\frac{0}{\frac{0}{4}}$ & 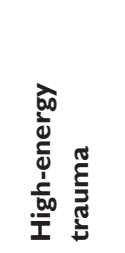 & 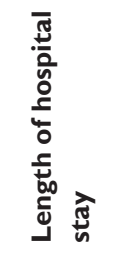 & 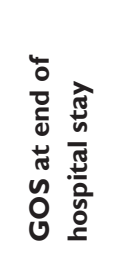 & 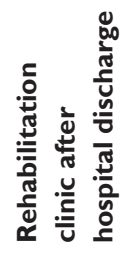 \\
\hline 0.03 & -0.05 & -0.03 & $-0.31^{* * * *}$ & -0.02 & $-0.22^{* * * *}$ & 0.00 & $-0.10^{* *}$ & $0.11 * *$ & -0.02 & $-0.37 * * *$ & $0.18^{* * *}$ & $-0.24 * * *$ \\
\hline 0.01 & 0.00 & -0.04 & $-0.2 * * *$ & 0.02 & $-0.20 * * *$ & 0.05 & $-0.15^{* * *}$ & $0.13^{* * *}$ & -0.04 & $-0.28 * * *$ & $0.19 * * *$ & $-0.29 * * *$ \\
\hline 0.04 & -0.03 & $-0 . I^{*}$ & $-0.22^{* * * *}$ & 0.02 & -0.01 & -0.03 & -0.06 & -0.03 & $-0.1 I^{* *}$ & $-0.20 * * *$ & 0.07 & $-0.17^{* * *}$ \\
\hline 0.04 & -0.04 & -0.05 & $-0.13 * * *$ & -0.02 & $-0.08^{*}$ & -0.02 & -0.05 & 0.06 & -0.04 & $-0.16 * * *$ & $0.10 * *$ & $-0.17^{* * *}$ \\
\hline 0.00 & 0.04 & 0.04 & $-0.08^{*}$ & -0.03 & -0.06 & 0.07 & $-0.08^{*}$ & $0.08^{*}$ & 0.01 & $-0.14 * * *$ & $0.11 * *$ & $-0.16^{* * *}$ \\
\hline-0.05 & 0.03 & 0.02 & $-0.10^{* *}$ & 0.01 & $-0.10^{* *}$ & $0.08^{*}$ & $-0.08^{*}$ & $0.11 * *$ & 0.00 & $-0.18 * * *$ & $0.10^{* *}$ & $-0.20 * * *$ \\
\hline-0.03 & 0.00 & 0.02 & -0.07 & 0.02 & $-0.15 * * *$ & $0.10^{* *}$ & $-0.12^{* * * *}$ & $0.13 * * *$ & 0.00 & $-0.17^{* * *}$ & $0.14^{* * *}$ & $-0.23 * * *$ \\
\hline-0.01 & 0.04 & 0.05 & $-0.08^{*}$ & 0.02 & -0.02 & 0.06 & -0.06 & 0.05 & -0.02 & $-0.14 * * *$ & 0.03 & $-0.11 * *$ \\
\hline $0.08^{*}$ & -0.06 & $-0.10 * *$ & $-0.29 * * * *$ & -0.01 & $-0.18^{* * *}$ & -0.02 & $-0.10^{* *}$ & 0.07 & -0.06 & $-0.3 I^{* * * *}$ & $0.19 * * *$ & $-0.24 * * *$ \\
\hline-0.03 & 0.06 & 0.07 & 0.01 & 0.03 & -0.07 & $0.13^{* * * *}$ & $-0.09 *$ & $0.12^{* *}$ & 0.04 & $-0.09 *$ & $0.10^{* *}$ & $-0.15^{* * *}$ \\
\hline-0.04 & 0.03 & 0.01 & $-0.07^{*}$ & -0.01 & -0.01 & 0.05 & $-0.10 * *$ & 0.02 & 0.00 & $-0.12 * *$ & 0.02 & $-0.12^{* * *}$ \\
\hline-0.05 & 0.04 & -0.03 & -0.03 & -0.02 & 0.01 & 0.01 & $-0.1 I^{* *}$ & 0.00 & 0.00 & $-0.14 * * *$ & 0.00 & $-0.13^{* * *}$ \\
\hline 0.02 & 0.06 & -0.06 & $-0.09 *$ & -0.04 & 0.07 & -0.05 & -0.01 & -0.07 & 0.00 & $-0.12 * *$ & -0.07 & -0.01 \\
\hline 0.00 & 0.00 & -0.04 & $-0.09 *$ & 0.01 & -0.05 & 0.07 & $-0.1 I^{* *}$ & 0.04 & -0.03 & $-0.15^{* * *}$ & 0.07 & $-0.13^{* * *}$ \\
\hline 0.01 & -0.05 & 0.03 & $-0.15^{* * * *}$ & -0.01 & $-0.17^{* * *}$ & 0.01 & $-0.1 I^{* *}$ & $0.11 * *$ & 0.02 & $-0.23 * * *$ & $0.12^{* *}$ & $-0.12^{* *}$ \\
\hline$-0.08 *$ & 0.02 & 0.03 & 0.00 & 0.01 & $-0.15 * * *$ & $0.14 * * *$ & $-0.17^{* * *}$ & $0.16 * * *$ & 0.01 & $-0.15^{* * *}$ & $0.15^{* * *}$ & $-0.23 * * *$ \\
\hline-0.05 & 0.02 & $-0.10 * *$ & $-0.12^{* *}$ & -0.07 & 0.06 & 0.00 & $-0.13^{* * *}$ & $-0.10 * *$ & -0.02 & $-0.24 * * *$ & 0.02 & -0.04 \\
\hline-0.04 & 0.01 & -0.01 & $-0.12^{* * * *}$ & 0.00 & -0.01 & $0.08^{*}$ & $-0.11 * *$ & 0.05 & -0.07 & $-0.16^{* * *}$ & $0.09 *$ & $-0.14^{* * *}$ \\
\hline 0.04 & 0.02 & 0.04 & 0.01 & 0.01 & $-0.10^{* *}$ & 0.03 & 0.04 & $0.10 * *$ & $0.11 * *$ & -0.02 & -0.04 & 0.02 \\
\hline 0.02 & 0.03 & 0.03 & -0.02 & -0.04 & -0.07 & 0.00 & 0.04 & $0.08 *$ & $0.11 * *$ & -0.01 & -0.05 & 0.03 \\
\hline 0.03 & 0.00 & -0.03 & $-0.16 * * *$ & -0.02 & 0.00 & -0.02 & -0.05 & -0.01 & -0.04 & $-0.14^{* * *}$ & -0.02 & -0.07 \\
\hline-0.02 & -0.03 & -0.02 & $-0.16 * * *$ & -0.01 & -0.06 & 0.05 & $-0.09 *$ & 0.06 & -0.03 & $-0.15^{* * *}$ & $0.09 *$ & $-0.12^{* *}$ \\
\hline-0.01 & -0.02 & -0.06 & $-0.18^{* * * *}$ & -0.03 & $0.07^{*}$ & -0.04 & $-0.09 *$ & $-0.09 *$ & $-0.14 * * *$ & $-0.13^{* * *}$ & 0.01 & $-0.09 *$ \\
\hline-0.03 & -0.05 & -0.05 & $-0.16 * * *$ & 0.02 & -0.02 & 0.06 & $-0.13 * * *$ & 0.01 & $-0.1 I^{* *}$ & $-0.15 * * *$ & $0.13^{* * * *}$ & $-0.14 * * *$ \\
\hline 0.03 & 0.00 & 0.04 & $0.13^{* * * *}$ & -0.01 & 0.07 & -0.02 & $0.10 * *$ & -0.03 & 0.02 & $0.22^{* * *}$ & -0.05 & $0.15 * * *$ \\
\hline-0.01 & -0.02 & -0.04 & $-0.19 * * *$ & -0.03 & -0.06 & 0.01 & $-0.13 * * *$ & 0.02 & -0.02 & $-0.25 * * *$ & 0.06 & $-0.11 * *$ \\
\hline-0.04 & 0.03 & -0.02 & $-0.08^{*}$ & -0.01 & -0.04 & 0.07 & $-0.13 * * *$ & 0.05 & -0.02 & $-0.18 * * *$ & 0.06 & $-0.17^{* * *}$ \\
\hline-0.03 & 0.05 & 0.05 & -0.01 & 0.03 & $-0.13^{* * *}$ & $0.13 * * *$ & $-0.10 * *$ & $0.18^{* * *}$ & 0.03 & $-0.13^{* * *}$ & $0.11 * *$ & $-0.19 * * *$ \\
\hline 0.01 & 0.01 & 0.01 & $-0.09 *$ & 0.04 & -0.04 & $0.09 *$ & $-0.09 *$ & $0.09 *$ & -0.02 & $-0.20 * * *$ & $0.09 *$ & $-0.19 * * *$ \\
\hline-0.01 & 0.01 & -0.01 & $-0.14 * * *$ & 0.01 & $-0.18 * * *$ & $0.14 * * *$ & $-0.15^{* * *}$ & $0.17^{* * *}$ & 0.00 & $-0.27 * * *$ & $0.18^{* * * *}$ & $-0.24 * * *$ \\
\hline 0.02 & -0.06 & -0.02 & -0.04 & 0.02 & -0.03 & $0.09 *$ & $-0.1 I^{* *}$ & 0.07 & -0.04 & $-0.12 * *$ & 0.07 & $-0.14 * * *$ \\
\hline-0.06 & 0.00 & -0.03 & -0.06 & -0.05 & 0.01 & 0.02 & -0.10 ** & 0.01 & -0.05 & $-0.10 * *$ & 0.03 & $-0.12^{* * *}$ \\
\hline-0.04 & -0.01 & -0.03 & $-0.19 * * * *$ & -0.05 & $-0.18 * * *$ & 0.07 & $-0.13 * * *$ & $0.12^{* * *}$ & -0.03 & $-0.25 * * *$ & 0.12 ** & $-0.25 * * *$ \\
\hline-0.03 & 0.02 & 0.01 & $-0.09 *$ & 0.01 & $-0.12 * * *$ & $0.12^{* *}$ & $-0.13 * * *$ & $0.15^{* * *}$ & -0.01 & $-0.21 * * *$ & $0.13 * * *$ & $-0.23 * * *$ \\
\hline-0.01 & -0.02 & -0.06 & $-0.25 * * *$ & -0.01 & $-0.10^{*}$ & -0.01 & $-0.10^{*}$ & 0.03 & -0.06 & $-0.31 * * *$ & $0.12^{* *}$ & $-0.2^{* * *}$ \\
\hline-0.05 & 0.01 & -0.01 & $-0.18 * * *$ & 0.04 & $-0.21 * * *$ & $0.09 *$ & $-0.12^{* *}$ & $0.10 * *$ & -0.04 & $-0.23 * * *$ & $0.21^{* * *}$ & $-0.17^{* * *}$ \\
\hline-0.03 & -0.03 & 0.00 & $-0.22^{* * * *}$ & -0.05 & $-0.10^{*}$ & $0.19 * * *$ & $-0.24 * * *$ & $0.18^{* * *}$ & -0.08 & $-0.37 * * *$ & $0.22^{* * *}$ & $-0.24 * * *$ \\
\hline
\end{tabular}


Gross et al

Dovepress

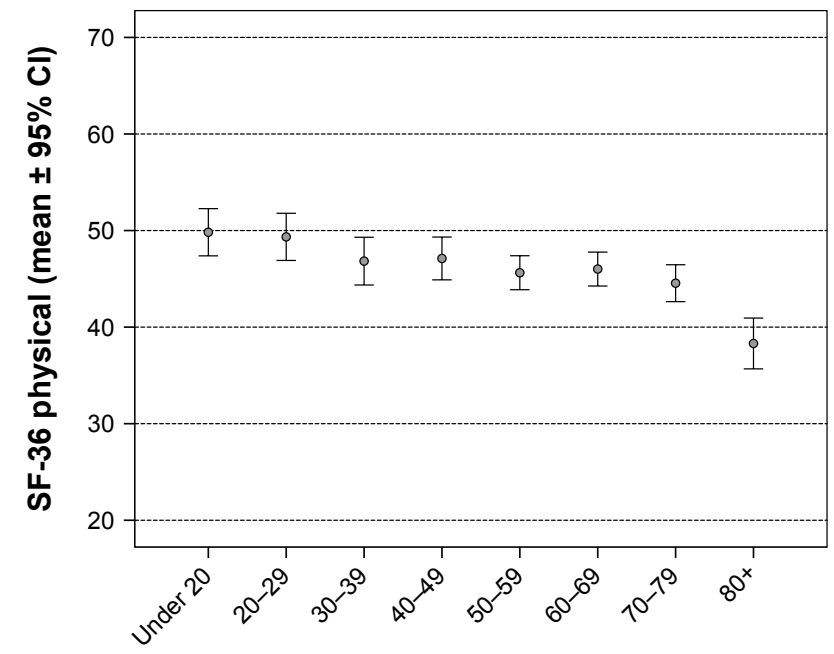

Age (years)

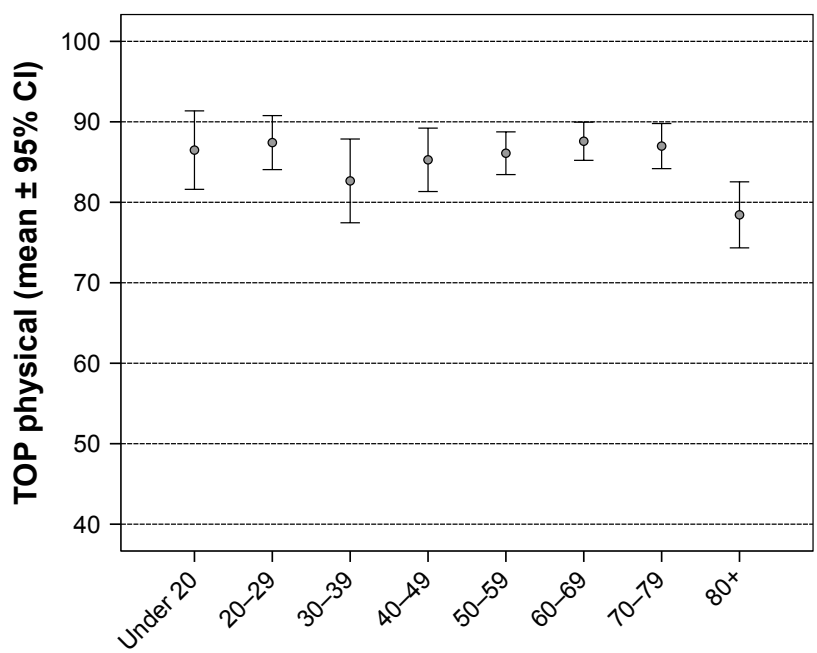

Age (years)

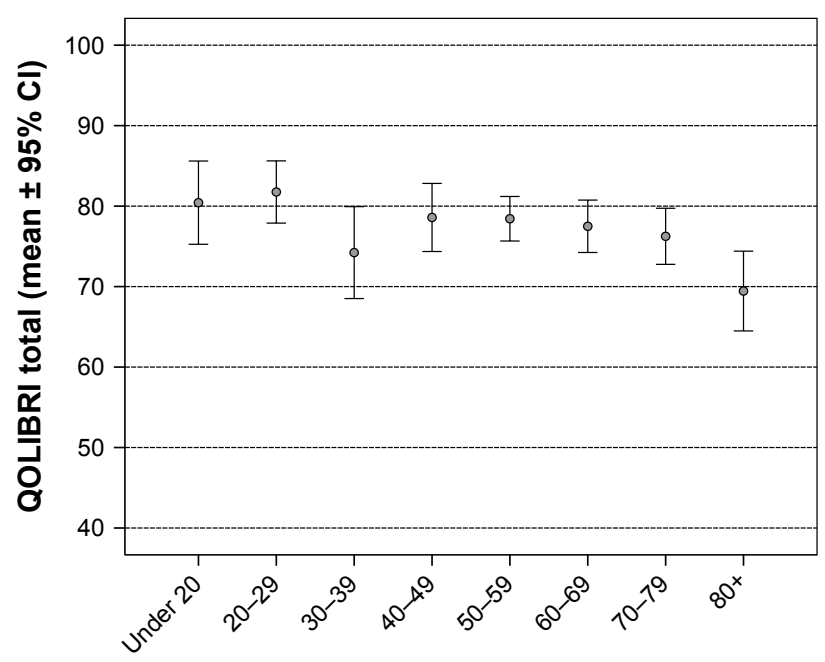

Age (years)

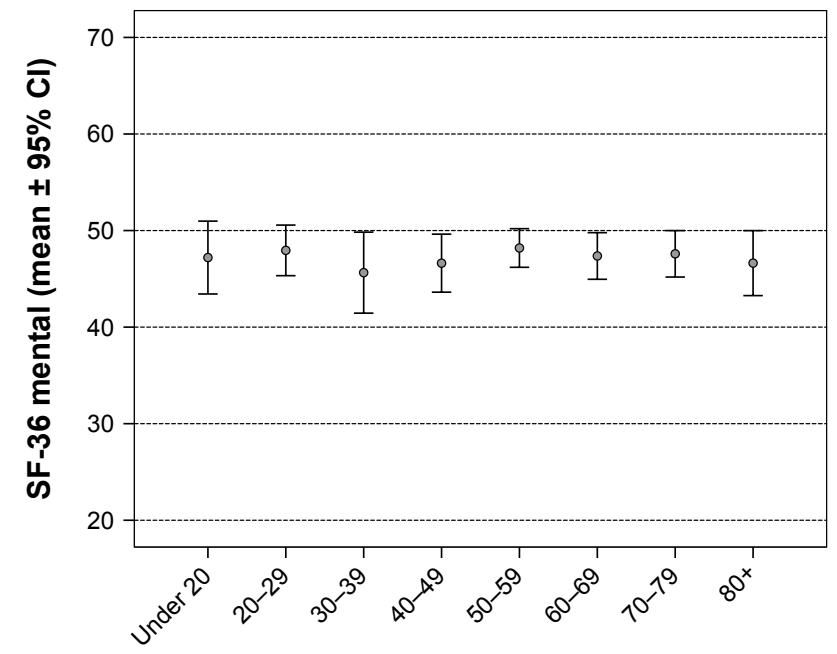

Age (years)


Age (years)

Figure 2 (Continued)

780

submit your manuscript | www.dovepress.com

Clinical Interventions in Aging 2018:13 


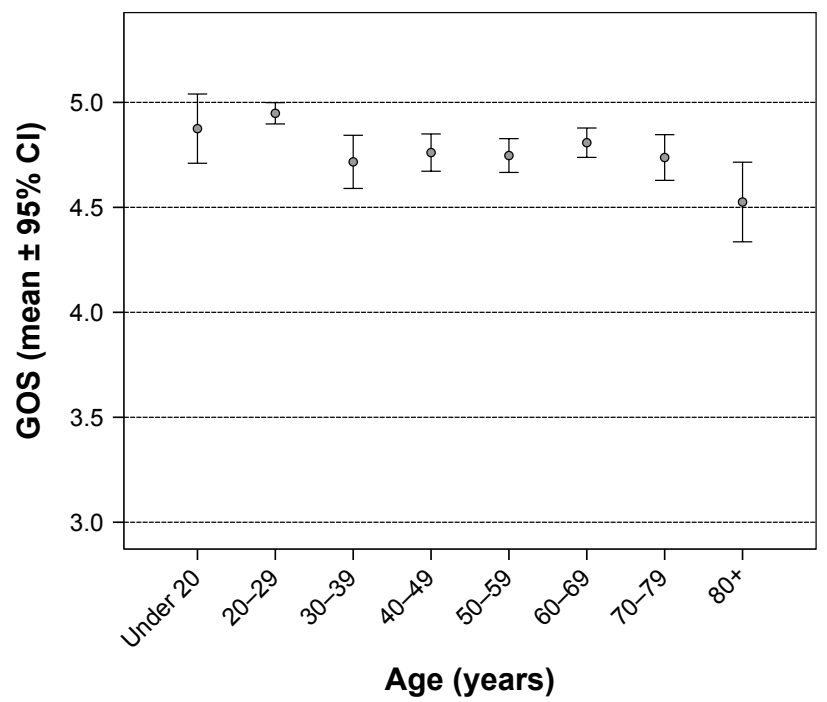

\begin{tabular}{|c|l|}
\hline Age groups (years) & $\mathbf{N}(\%)$ \\
\hline Under 20 & $40(5.6)$ \\
\hline $20-29$ & $79(11)$ \\
\hline $30-39$ & $61(8.5)$ \\
\hline $40-49$ & $93(13)$ \\
\hline $50-59$ & $155(21.6)$ \\
\hline $60-69$ & $128(17.8)$ \\
\hline $70-79$ & $103(14.3)$ \\
\hline $80+$ & $59(8.2)$ \\
\hline Total & $\mathbf{7 1 8 ( 1 0 0 )}$ \\
\hline
\end{tabular}

Figure 2 Longer-term outcomes in single scores for different age groups.

Abbreviations: SF-36, trauma medical outcomes study Short Form-36 (physical and mental sum component); EQ-5D, Euro Quality of Life Group health-related quality of life on five dimensions; GOS, Glasgow Outcome Scale; TOP, Trauma Outcome Profile (physical and mental component); QOLIBRI, Quality of Life after Brain Injury.

component of the TOP. ${ }^{33}$ The only other study analyzing a possible correlation of age with the longer-term outcome (at mean 2.7 years) as measured in the TOP for polytrauma patients (mean ISS 27) found a maximum marginal effect in single dimensions, largest for social interaction (Pearson $r=0.25) .{ }^{11}$ In a longer-term outcome study on 355 patients 1-5 years following severe trauma (mean ISS 24) and using the SF-36 only, Harris et $\mathrm{al}^{34}$ reported a correlation with the PCS and a trend for the MCS in univariate analysis, but in the subsequent multivariate analysis the PCS was no longer significant and a lower MCS was associated with decreasing age. In a longitudinal 2-year follow-up study of severe trauma (ISS $>15$ ) in adult patients up to the age of 67 years, using the SF-36, the World Health Organization Disability Schedule II, and cognitive function questions, Soberg et $\mathrm{al}^{16}$ found that age was not a significant predictor of outcome in multivariate analysis. Kiely et al came to the same conclusion following a multivariate analysis in a longitudinal study up to 6 months following major trauma (ISS >8) using the SF-36. ${ }^{15}$ With regard to return to work, a longitudinal study on the 2-year follow-up of 97 trauma patients (NISS $>15$; 18-67 years old) revealed no correlation with age in a univariate analysis. ${ }^{35}$ In addition, in 115 adult polytrauma patients (mean ISS 27 , median 2.5 years after injury), no association of age with working capacity was found in a multivariate analysis. ${ }^{36}$

Comparing younger major trauma (ISS >15) patients aged $20-30$ years with those aged $\geq 60$ years, no differences with regard to the 2-year outcome as measured with the
GOS were found in a study in the Netherlands. ${ }^{37}$ A Canadian trauma center phone survey compared the outcome of the injured (mean ISS 21 ) aged $\geq 65$ years with age-adjusted norm values in the SF-36 and revealed significantly lower values of the injured in seven of eight domains at a mean of 2.8 years following trauma. No difference was observed between the elderly injured and non-injured of comparable age with regard to their level of bodily pain or daily activities. ${ }^{38}$ A German national health survey in persons between 18 and 80 years demonstrated a decrease with increasing age in a person's self-assessment of physical activity, but not in social or psychological dimensions of the SF-36. ${ }^{39}$ With regard to the EuroQoL, Szende and Williams reported a continuous decrease in the mean EuroQoL visual analog scale (EQ-VAS) with increasing age for single decennia from 18 years up to $80+$ years..$^{28}$ The rate of self-reported problems on the five dimensions of EQ-5D was shown to be highly variable between countries with the resulting mean EQ-VAS values varying between 67 and 83 (scale 0-100). As such, any comparison of bare values in the literature has to be undertaken very carefully, given the presumably crosscultural impact of differences observed. To our knowledge, for the TOP and the QOLIBRI no detailed normative data with regard to differences between age groups are currently available.

At present, these conflicting results appear difficult to explain. But as discussed, most of the studies reporting on an important (negative) correlation of age with longer-term 
Table 4 Sociodemographics, trauma, and treatment characteristics of major trauma patients aged $\geq 80$ years vs younger patients

\begin{tabular}{|c|c|c|c|}
\hline \multirow[t]{2}{*}{ Variable } & \multirow{2}{*}{$\begin{array}{l}<80 \text { years of age } \\
(\mathrm{N}=659) \\
\text { Mean }(\mathrm{SD})\end{array}$} & \multirow{2}{*}{$\begin{array}{l}\geq 80 \text { years } \\
(\mathrm{N}=59) \\
\text { Mean }(\mathrm{SD})\end{array}$} & \multirow[t]{2}{*}{$p$-value } \\
\hline & & & \\
\hline $\begin{array}{l}\text { Age at time of injury } \\
\text { (years) }\end{array}$ & $50.67(17.69)$ & $84.44(3.97)$ & $<0.001$ \\
\hline GCS & $13.5(3.16)$ & 14.39 (I.2) & 0.031 \\
\hline MGAP & $25.26(4.03)$ & $23.22(1.48)$ & $<0.001$ \\
\hline ISS & I3.86 (7.52) & 13.14 (7.19) & 0.475 \\
\hline NISS & $18.48(9.28)$ & $17.24(8.34)$ & 0.320 \\
\hline RISC 2 & $2.7(5.27)$ & I 3.55 (13.04) & $<0.001$ \\
\hline SAPS II & $26.35(11)$ & $37.21(10.01)$ & $<0.001$ \\
\hline AIS I head and neck & I.7I (1.52) & $1.54(1.69)$ & 0.425 \\
\hline AIS 2 face & $0.4 I(0.86)$ & $0.32(0.73)$ & 0.473 \\
\hline AIS 3 chest & $1.07(1.44)$ & $1.07(1.38)$ & 0.979 \\
\hline AIS 4 abdomen & $0.49(1.04)$ & $0.4 I(0.87)$ & 0.542 \\
\hline AIS 5 extremities & $1.22(1.28)$ & $\mathrm{I} .24(\mathrm{I} .4 \mathrm{I})$ & 0.942 \\
\hline AIS 6 external & $0.38(0.56)$ & $0.36(0.52)$ & 0.726 \\
\hline $\begin{array}{l}\text { Length of hospital } \\
\text { stay (days) }\end{array}$ & $12.77(12)$ & $15.39(10.04)$ & 0.104 \\
\hline \multirow{2}{*}{$\begin{array}{l}\text { GOS (at hospital } \\
\text { discharge) }\end{array}$} & $4.85(0.4)$ & $4.83(0.38)$ & 0.639 \\
\hline & $n(n \%)$ & $n(n \%)$ & $p$-value \\
\hline Gender, female & $212(32.2)$ & $27(45.8)$ & 0.034 \\
\hline Swiss & $538(83.8)$ & $58(100)$ & 0.001 \\
\hline Compulsory & $106(16.3)$ & $18(30.5)$ & \\
\hline education & $430(66.3)$ & $36(61.0)$ & 0.004 \\
\hline $\begin{array}{l}\text { Professional } \\
\text { qualification }\end{array}$ & $113(17.4)$ & $5(8.5)$ & \\
\hline University degree & & & \\
\hline Living in a partnership & $356(54.4)$ & $30(50.8)$ & 0.898 \\
\hline High-energy trauma & $369(56.1)$ & $22(37.3)$ & 0.005 \\
\hline $\begin{array}{l}\text { Need for emergency } \\
\text { operation }\end{array}$ & $50(7.6)$ & $3(5.1)$ & 0.482 \\
\hline ICU stay & $357(54.2)$ & $28(47.5)$ & 0.322 \\
\hline ISS $>16$ & $231(35.1)$ & $19(32.2)$ & 0.660 \\
\hline Multiply injured & $460(69.8)$ & $36(6 I)$ & 0.162 \\
\hline $\begin{array}{l}\text { Rehabilitation clinic } \\
\text { following initial } \\
\text { hospital stay }\end{array}$ & $196(29.7)$ & $27(45.8)$ & 0.011 \\
\hline
\end{tabular}

Abbreviations: GCS, Glasgow Coma Scale; MGAP, Mechanism, Glasgow Coma Scale, Age, and Arterial score; ISS, Injury Severity Score; NISS, New Injury Severity Score; RISC 2, Revised Injury Severity Classification 2; SAPS II, Simplified Acute Physiology Score II; AIS, Abbreviated Injury Scale (eg, AIS I: head and neck etc); GOS, Glasgow Outcome Scale; ICU, intensive care unit, multiply injured.

outcome only undertook nonadjusted univariate analyses. None of the evaluations additionally using multivariate analysis found such an association or at maximum only a discrete effect. Our prospective investigation, the first evaluating this topic in a meticulous manner by using a well-defined study cohort of trauma patients and comparing various measures of longer-term outcome, even within separated decennia and including multivariate analysis, may describe the missing cornerstone in the context: the group of "at least octogenarians" apparently explain what was earlier interpreted as a general effect of age on outcome.

\section{Limitations}

Until confirmed in further studies the presented results are restricted to the limited number of patients in this monocenter cohort and the definition of major trauma used. Given the comparable outcomes for patients at 1- and 2-year follow-ups found in earlier evaluations of our own data and the literature, ${ }^{16}$ longer-term outcome was defined for this investigation as at least a 1-year outcome. Even though differences in the characteristics of age groups analyzed in more detail were only minor and multivariate analysis was used to exclude possible interactions, all conclusions are confined to the variables tested. Although this investigation included various measures of outcome, given the objective to compare QoL across all adult age groups, scores specifically developed for the elderly ${ }^{5,40}$ were not implemented. The complexity of the questionnaire used was already on the upper reasonable limit. Similarly, specific subtopics regarding the interpretation of QoL depending on the perspectives of differing age groups or the particularly inclusion of frailty ${ }^{5,6,41}$ in the context of major trauma await further clarification in subsequent studies. In the literature, the definition of the elderly varies widely, partly starting at an age of $55^{17}$ or at least 65 years. ${ }^{3}$ By evaluating single decennia in addition to unstratified analysis we were able to substantiate the group of at least octogenarians in this context. From a statistical point of view, the way to handle single missing data can be discussed. The imputation method, that is, replacing missing values by the mean of the sample, as used in this investigation for multivariate regression analysis is standardly used in the literature instead of listwise exclusion of whole cases ${ }^{42}$ thereby accepting the corresponding risk of inaccuracy of data ${ }^{43}$ as a minor risk. In this investigation, the maximum number of missing values was $28(<4 \%)$ for the SF-36. As with many outcome studies in trauma, our response rate of $44 \%$ is low, though within the range of the literature, $7,15,16,33,44$ especially if seen in the light of the fact that respondents for this investigation had to answer an extensive postal questionnaire in contrast to studies using only one or two outcome measures or telephone interviews. It is well known that response rates tend to decrease with the extent of questionnaires and with the time after injury. ${ }^{12}$ For example, phone interviews may result in higher response rates, especially if few questions and short scores are used, but this procedure has its own limitations and other selection biases. ${ }^{45-47}$ As was pointed out by Zelle et al, ${ }^{48}$ we clearly stated the loss of follow-up and did not artificially exclude several subgroups of 
Table 5 Multivariate analysis (stepwise regression analysis) of demographics, trauma, and treatment characteristics of longer-term outcomes based on scores and capacity to work for all major trauma patients vs those under the age of 80 years

\begin{tabular}{|c|c|c|c|c|c|c|c|c|c|c|}
\hline \multirow[t]{2}{*}{ Variables } & \multicolumn{5}{|c|}{ All patients $(\mathrm{N}=7 \mid 8)$} & \multicolumn{5}{|c|}{ Patients $<80$ years of age $(\mathrm{N}=659)$} \\
\hline & Beta & p-value & Total $\mathbf{R}^{2}$ & $\mathbf{R}^{2}$ change & p change & Beta & p-value & Total $\mathbf{R}^{2}$ & $\mathbf{R}^{2}$ change & $p$ change \\
\hline \multicolumn{11}{|c|}{ Dependent: SF-36 physical } \\
\hline AIS 5 extremities & -0.24 & $<0.00$ I & 0.08 & 0.08 & $<0.001$ & -0.27 & $<0.001$ & 0.08 & 0.08 & $<0.001$ \\
\hline Age at time of injury & -0.20 & $<0.00$ I & 0.13 & 0.06 & $<0.001$ & -0.17 & $<0.001$ & 0.10 & 0.03 & $<0.001$ \\
\hline AIS 4 abdomen & -0.07 & 0.041 & 0.14 & 0.01 & 0.005 & -0.11 & 0.003 & 0.12 & 0.01 & 0.004 \\
\hline Educational level & 0.09 & 0.010 & 0.15 & 0.01 & 0.009 & 0.09 & 0.011 & 0.12 & 0.01 & 0.011 \\
\hline RISC 2 & -0.12 & 0.003 & 0.16 & 0.01 & 0.021 & & & & & \\
\hline AIS I head and neck & 0.10 & 0.022 & 0.17 & 0.01 & 0.022 & & & & & \\
\hline \multicolumn{11}{|c|}{ Dependent: SF-36 mental } \\
\hline Educational level & 0.18 & $<0.00 \mathrm{I}$ & 0.03 & 0.03 & $<0.001$ & 0.18 & $<0.00 \mathrm{I}$ & 0.04 & 0.04 & $<0.001$ \\
\hline AIS I head and neck & -0.12 & 0.001 & 0.05 & 0.01 & 0.001 & -0.11 & 0.005 & 0.05 & 0.01 & 0.005 \\
\hline \multicolumn{11}{|c|}{ Dependent: TOP physical } \\
\hline AIS 5 extremities & -0.19 & $<0.00 \mathrm{I}$ & 0.04 & 0.04 & $<0.001$ & & & & & \\
\hline Educational level & 0.18 & $<0.00 \mathrm{I}$ & 0.07 & 0.03 & $<0.001$ & 0.20 & $<0.001$ & 0.04 & 0.04 & $<0.001$ \\
\hline \multicolumn{11}{|c|}{ Dependent: TOP mental } \\
\hline Educational level & 0.20 & 0.000 & 0.05 & 0.05 & $<0.001$ & 0.22 & $<0.00$ I & 0.05 & 0.05 & $<0.001$ \\
\hline AIS 5 extremities & -0.12 & 0.002 & 0.05 & 0.01 & 0.038 & & & & & \\
\hline AIS I head and neck & -0.12 & 0.003 & 0.06 & 0.01 & 0.003 & & & & & \\
\hline \multicolumn{11}{|c|}{ Dependent: QOLIBRI total } \\
\hline Educational level & 0.19 & $<0.00$ I & 0.04 & 0.04 & $<0.001$ & 0.19 & $<0.001$ & 0.04 & 0.04 & $<0.00$ I \\
\hline Age at time of injury & -0.12 & 0.001 & 0.05 & 0.01 & 0.001 & & & & & \\
\hline AIS 5 extremities & -0.12 & 0.002 & 0.06 & 0.01 & 0.026 & -0.13 & 0.001 & 0.05 & 0.01 & 0.025 \\
\hline AIS I head and neck & -0.11 & 0.006 & 0.07 & 0.01 & 0.006 & -0.12 & 0.006 & 0.06 & 0.01 & 0.006 \\
\hline \multicolumn{11}{|c|}{ Dependent: GOS at longer term* } \\
\hline RISC 2/AIS 5 extremities & -0.17 & $<0.00 \mathrm{I}$ & 0.04 & 0.04 & $<0.001$ & -0.16 & $<0.00 \mathrm{I}$ & 0.03 & 0.03 & $<0.001$ \\
\hline AIS 5 extremities/RISC 2 & -0.18 & $<0.00 \mathrm{I}$ & 0.07 & 0.03 & $<0.001$ & -0.15 & $<0.00 \mathrm{I}$ & 0.05 & 0.02 & $<0.001$ \\
\hline Age at time of injury & -0.08 & 0.044 & 0.08 & 0.01 & 0.044 & & & & & \\
\hline \multicolumn{11}{|l|}{ Dependent: EuroQoL } \\
\hline AIS 5 extremities & -0.24 & $<0.001$ & 0.06 & 0.06 & $<0.001$ & -0.24 & $<0.001$ & 0.06 & 0.06 & $<0.001$ \\
\hline Educational level & 0.16 & $<0.00 \mathrm{I}$ & 0.09 & 0.03 & $<0.001$ & 0.16 & $<0.001$ & 0.08 & 0.02 & $<0.001$ \\
\hline Age at time of injury & -0.16 & $<0.00 \mathrm{I}$ & 0.11 & 0.02 & $<0.001$ & -0.10 & 0.007 & 0.09 & 0.01 & 0.007 \\
\hline \multicolumn{11}{|c|}{ Employed patients $<64$ years $(\mathrm{N}=383)$} \\
\hline \multicolumn{11}{|c|}{ Dependent: working capacity at longer term } \\
\hline AIS 5 extremities & -0.28 & $<0.001$ & 0.05 & 0.05 & $<0.001$ & & & & & \\
\hline AIS I head and neck & -0.17 & 0.002 & 0.07 & 0.03 & 0.001 & & & & & \\
\hline Age at time of injury & -0.18 & $<0.001$ & 0.10 & 0.02 & 0.002 & & & & & \\
\hline Educational level & 0.14 & 0.004 & 0.12 & 0.02 & 0.004 & & & & & \\
\hline
\end{tabular}

Note: *Order of entry differed for the study group of all patients: step I = RISC, step 2 = AIS 5 vs for patients $<80$ years of age only: step I = AIS 5 , step 2 = RISC. Abbreviations: AIS, Abbreviated Injury Scale (eg, AIS I: head and neck etc); RISC 2, Revised Injury Severity Classification 2; SF-36, trauma medical outcomes study Short Form-36, physical and mental sum component score; TOP, Trauma Outcome Profile, physical and mental component; QOLIBRI, Quality of Life after Brain Injury total score; GOS, Glasgow Outcome Scale; EuroQoL, Euro Quality of Life.

patients whose responses were difficult to obtain. In addition, respondents did not differ from nonrespondents in major criteria such as mean age, gender, or overall trauma severity.

\section{Conclusion}

This pilot study for the first time demonstrated that, if at all, the small impact of age on the longer-term outcomes of major trauma patients overall appears only to be relevant "after fourscore," that is, in at least octogenarians. If affirmed by other study groups, this finding has to be taken into account for all resuscitation efforts as well as rehabilitation strategies. It is proposed that more work should be done to develop accordant clinical pathways and guidelines.

\section{Acknowledgments}

This study was financially supported by the Research Fund of the hospital. The authors are grateful for the commitment of L Meier, F Maeder, and M Keller in collecting original 
data, the editorial assistance of J Buchanan, and thank all the patients and hospital collaborators involved in the study.

\section{Disclosure}

The authors report no conflicts of interest in this work.

\section{References}

1. Hammer PM, Storey AC, Bell T, et al. Improving geriatric trauma outcomes: a small step toward a big problem. J Trauma Acute Care Surg. 2016;81(1):162-167.

2. Follin A, Jacqmin S, Chhor V, et al. Tree-based algorithm for prehospital triage of polytrauma patients. Injury. 2016;47(7):1555-1561.

3. Caterino JM, Brown NV, Hamilton MW, et al. Effect of geriatric-specific trauma triage criteria on outcomes in injured older adults: a statewide retrospective cohort study. J Am Geriatr Soc. 2016;64(10):1944-1951.

4. Shipman KE, Doyle A, Arden H, Jones T, Gittoes NJ. Development of fracture liaison services: what have we learned? Injury. 2017; 48(Suppl 7):S4-S9.

5. Milte R, Crotty M. Musculoskeletal health, frailty and functional decline. Best Pract Res Clin Rheumatol. 2014;28(3):395-410.

6. Brooks SE, Peetz AB. Evidence-based care of geriatric trauma patients. Surg Clin North Am. 2017;97(5):1157-1174.

7. Rainer TH, Yeung HH, Gabbe BJ, et al. A comparison of functional outcome in patients sustaining major trauma: a multicentre, prospective, international study. PLoS One. 2014;9(8):e103396.

8. Gunning A, van Heijl M, van Wessem K, Leenen L. The association of patient and trauma characteristics with the health-related quality of life in a Dutch trauma population. Scand J Trauma Resusc Emerg Med. 2017;25(1):41.

9. Fayers PM, Hand DJ, Bjordal K, Groenvold M. Causal indicators in quality of life research. Qual Life Res. 1997;6(5):393-406.

10. Poolman RW, Swiontkowski MF, Fairbank JCT, Schemitsch EH, Sprague S, de Vet HCW. Outcome instruments: rationale for their use. J Bone Joint Surg Am. 2009;91(Suppl 3):41-49.

11. Attenberger C, Amsler F, Gross T. Clinical evaluation of the Trauma Outcome Profile (TOP) in the longer-term follow-up of polytrauma patients. Injury. 2012;43(9):1566-1574.

12. Holbrook TL, Anderson DJ, Sieber WJ, Browner BD, Hoyt DB. Outcome after major trauma - 12-month and 18-month follow-up results from the Trauma Recovery Project. J Trauma. 1999;46(5):765-773.

13. van Delft-Schreurs CCHM, van Son MAC, de Jongh MAC, Lansink KWW, de Vries J, Verhofstad MHJ. The relationship between physical and psychological complaints and quality of life in severely injured patients. Injury. 2017;48(9):1978-1984.

14. Sluys K, Häggmark T, Iselius L. Outcome and quality of life 5 years after major trauma. $J$ Trauma. 2005;59(1):223-232.

15. Kiely JM, Brasel KJ, Weidner KL, Guse CE, Weigelt JA. Predicting quality of life six months after traumatic injury. $J$ Trauma. 2006; 61(4):791-798.

16. Soberg HL, Bautz-Holter E, Roise O, Finset A. Long term multidimensional functional consequences of severe multiple injuries two years after trauma: a prospective longitudinal cohort study. $J$ Trauma. 2007;62(2):461-470.

17. Newgard CD, Richardson D, Holmes JF, et al. Physiologic field triage criteria for identifying seriously injured older adults. Prehosp Emerg Care. 2014;18(4):461-470.

18. Sutherland AG, Johnston AT. The new injury severity score: better prediction of functional recovery after musculoskeletal injury. Value Health. 2006;9(1):24-27.

19. American College of Surgeons Committee. Advanced Trauma Life Support (ATLS) Student Course Manual. Vol 9. Chicago, USA: American College of Surgeons; 2012.

20. Neugebauer EAM, Waydhas C, Lendemans S, Rixen D, Eikermann M, Pohlemann T. The treatment of patients with severe and multiple traumatic injuries. Dtsc Arztebl Int. 2012;109(6):102-108.
21. Jennett B, Bond M. Assessment of outcome after severe brain damage. Lancet. 1975;1(7905):480-484.

22. Teasdale G, Jennett B. Assessment and prognosis of coma after head injury. Acta Neurochir (Wien). 1976;34(1-4):45-55.

23. Sartorius D, Manach YL, David JS, et al. Mechanism, Glasgow coma scale, age, and arterial pressure (MGAP): a new prehospital triage to predict mortality in trauma patients. Crit Care Med. 2010;38(3): 831-837.

24. Le Gall JR, Lemeshow S, Saulnier F. A new simplified acute physiology score (SAPS II) based on a European/North American multicenter study. JAMA. 1993;270(24):2957-2963.

25. Gennarelli T. The Abbreviated Injury Scale - 1990 Revision. Des Plaines, IL: American Association for the Advancemnent of Automotive Medicine (AAAM); 1990.

26. Baker SP, O'Neill B, Haddon W, Long WB. The injury severity score: a method for describing patients with multiple injuries and evaluating emergency care. J Trauma. 1974;14(3):187-196.

27. Lefering R. Ein neuer Prognose-Score im TraumaRegister DGU (RISC II) [A new prognostic score in the TraumaRegister DGU (RISC II)]. Orthopädie und Unfallchirurgie Mitteilungen und Nachrichten. 2015:80-82. German.

28. Szende A, Williams A. Measuring Self-Reported Population Health: An International Perspective Based on EQ-5D. Budapest: Springer Med Publishing Ltd; 2004.

29. Bullinger M. German translation and psychometric testing of the SF-36 health survey: preliminary results from the IQOLA project. Soc Sci Med. 1995;41(10):1359-1366.

30. Pirente N, Gregor A, Bouillon B, Neugebauer E. Lebensqualität schwerstverletzter Patienten ein Jahr nach dem Trauma [Quality of life of severely injured patients one year after trauma. A matched-pair study with a healthy control group]. Unfallchirurg. 2001;104(1):57-63. German.

31. von Steinbuechel N, Wilson L, Gibbons H, et al. Quality of life after brain injury (QOLIBRI): scale validity and correlates of quality of life. J Neurotrauma. 2010;27(7):1157-1165.

32. von Steinbuechel N, Wilson L, Gibbons H, et al. Quality of life after brain injury (QOLIBRI): scale development and metric properties. J Neurotrauma. 2010;27(7):1167-1185.

33. Zwingmann J, Hagelschuer P, Langenmair E, et al. Lower health-related quality of life in polytrauma patients: long-term follow-up after over 5 years. Medicine. 2016;95(19):e3515.

34. Harris IAF, Young JMP, Rae HM, Jalaludin BBP, Solomon MJF. Predictors of general health after major trauma. J Trauma. 2008;64(4): 969-974.

35. Soberg HL, Finset A, Bautz-Holter E, Sandvik L, Roise O. Return to work after severe multiple injuries: a multidimensional approach on status 1 and 2 years postinjury. J Trauma. 2007;62(2):471-481.

36. Gross T, Attenberger C, Huegli RW, Amsler F. Factors associated with reduced longer-term capacity to work in patients following polytrauma-a Swiss trauma center experience. J Am Coll Surg. 2010;211(1):81-91.

37. van der Sluis CK, Klasen HJ, Eisma WH, ten Duis HJ. Major trauma in young and old: what is the difference? J Trauma. 1996;40(1):78-82.

38. Soberg HL, Roe C, Brunborg C, von Steinbuechel N, Andelic N. The Norwegian version of the QOLIBRI - a study of metric properties based on a 12 month follow-up of persons with traumatic brain injury. Health Qual Life Outcomes. 2017;15:14.

39. Ellert U, Bellach BM. Der SF-36 im Bundes-Gesundheitssurvey Beschreibung einer aktuellen Normstichprobe. Gesundheitswesen. 1999;61(Sonderheft 2):S184-S190. German.

40. Joseph B, Pandit V, Zangbar B, et al. Superiority of frailty over age in predicting outcomes among geriatric trauma patients: a prospective analysis. JAMA Surg. 2014;149(8):766-772.

41. Biffl WL, Biffl SE. Rehabilitation of the geriatric surgical patient: predicting needs and optimizing outcomes. Surg Clin North Am. 2015; 95(1):173-190. 
42. Sewalt CA, Wiegers EJA, Venema E, Lingsma HF. Letter to the Editor regarding the article: "identifying pre-hospital factors associated with outcome for major trauma patients in a regional trauma network: an exploratory study". Scand J Trauma Resusc Emerg Med. 2017; 25(1): 113 .

43. Heaney JB, Schroll R, Turney J, et al. Implications of the Trauma Quality Improvement Project inclusion of nonsurvivable injuries in performance benchmarking. J Trauma Acute Care Surg. 2017;83(4):617-621.

44. Weninger P, Aldrian S, Koenig F, Vecsei V, Nau T. Functional recovery at a minimum of 2 years after multiple injury - development of an outcome score. J Trauma. 2008;65(4):799-808.

45. MacKenzie EJ, McCarthy ML, Ditunno JF, et al. Using the SF-36 for characterizing outcome after multiple trauma involving head injury. J Trauma. 2002;52(3):527-534.
46. O'Cathain A, Knowles E, Nicholl J. Testing survey methodology to measure patients' experiences and views of the emergency and urgent care system: telephone versus postal survey. BMC Med Res Methodol. 2010;10(1):52.

47. Edwards PJ, Roberts I, Clarke MJ, et al. Methods to increase response to postal and electronic questionnaires. Cochrane Database Syst Rev. 2009;3:MR000008.

48. Zelle BA, Bhandari M, Sanchez AI, Probst C, Pape HC. Loss of follow-up in orthopaedic trauma: is $80 \%$ follow-up still acceptable? J Orthop Trauma. 2013;27(3):177-181.
Clinical Interventions in Aging

\section{Publish your work in this journal}

Clinical Interventions in Aging is an international, peer-reviewed journal focusing on evidence-based reports on the value or lack thereof of treatments intended to prevent or delay the onset of maladaptive correlates of aging in human beings. This journal is indexed on PubMed Central, MedLine,

\section{Dovepress}

CAS, Scopus and the Elsevier Bibliographic databases. The manuscript management system is completely online and includes a very quick and fair peer-review system, which is all easy to use. Visit http://www.dovepress. com/testimonials.php to read real quotes from published authors. 